\title{
Activity of Physalin F in a Collagen-Induced Arthritis Model
}

\author{
Daniele Brustolim, ${ }^{\dagger}$ Juliana F. Vasconcelos, ${ }^{\dagger}$ Luiz Antônio R. Freitas, ${ }^{\dagger}$ Mauro M. Teixeira, ${ }^{\ddagger}$ Marcel T. Farias, ${ }^{\dagger}$ \\ Yvone M. Ribeiro, ${ }^{\S}$ Therezinha C. B. Tomassini, ${ }^{\S}$ Geraldo G. S. Oliveira, ${ }^{\dagger}$ Lain C. Pontes-de-Carvalho, ${ }^{\dagger}$ \\ Ricardo Ribeiro-dos-Santos, ${ }^{\dagger, \perp}$ and Milena B. P. Soares ${ }^{*, \dagger, \perp}$ \\ Centro de Pesquisas Gonçalo Moniz, Fundação Oswaldo Cruz, Salvador, BA, Brazil, Universidade Federal de Minas Gerais, Belo Horizonte, \\ MG, Brazil, FarManguinhos, Fundação Oswaldo Cruz, Rio de Janeiro, RJ, Brazil, and Hospital São Rafael, Salvador, BA, Brazil
}

Received October 28, 2009

The effects of physalin F (1), a steroid derivative purified from Physalis angulata, were investigated in models of collagen-induced arthritis in DBA/1 mice and allergic airway inflammation in BALB/c mice. Oral treatment with 1 or dexamethasone caused a marked decrease in paw edema and joint inflammation when compared to vehicle-treated arthritic mice. In contrast, treatment with $\mathbf{1}$ had no effect in mice with allergic airway inflammation caused by ovalbumin immunization, whereas dexamethasone significantly reduced the number of inflammatory cells and eosinophils in the broncoalveolar lavage fluid and in lung sections of challenged mice. To further demonstrate that $\mathbf{1}$ acts through a mechanism different from that of glucocorticoids, a nuclear translocation assay was performed of the glucocorticoid receptor (GR) using COS-7 cells transfected with a plasmid encoding for a yellow fluorescent protein (YFP)-GR fusion protein. Untreated or treated cells with 1 had YFP staining mainly in the cytoplasm, whereas in dexamethasone-treated cells the YFP staining was concentrated in the nuclei. It is concluded that the mechanism of the immunosuppressive activity of physalin $\mathrm{F}$ is distinct from that of the glucocorticoids.

Immunosuppressive substances are used widely to inhibit undesired immune responses in autoimmune and allergic diseases, such as rheumatoid arthritis and asthma. Although several immunosuppressive drugs are currently available, their prolonged use is usually accompanied by undesired effects. An extensively utilized class of immunosuppressive agents is comprised by the glucocorticoids, which possess undesirable side effects, mainly associated with chronic use, such atherosclerosis, cataracts, fatty liver, growth retardation, osteoporosis, and skin atrophy. In addition, other side effects are common in patients with underlying risk factors, such as acne vulgaris, diabetes, hypertension, and peptic ulcers. ${ }^{1}$ Glucocorticoids act via glucocorticoid receptor (GR) activation, which mediates transactivation or transrepression of several genes, which explains their anti-inflammatory effect and, possibly, their adverse effects. ${ }^{2}$ Thus, the development of new immunosuppressors with different mechanisms of action and reduced toxic effects is of great interest.

Plant secondary metabolites are important for flavoring of food, for their resistance against pests, and as drugs, including substances having immunosuppressive activity. ${ }^{3,4}$ Examples of natural immunosuppressive compounds are lupeol and umbelliferone, which inhibit allergic airway inflammation in a mouse model. ${ }^{5,6}$ Ursolic $\operatorname{acid}^{7}$ and alkaloids from Radix linderae, ${ }^{8}$ the dry roots of Lindera aggregata (Sims) Kosterm., possess antiarthritic effects, as shown in experimental models of arthritis.

Physalis angulata L. (Solanaceae) is an herb widely used in popular medicine as an analgesic and antirheumatic and to treat sore throats and abdominal pain. It is considered as an antidiuretic, an anti-inflammatory for hepatitis and cervicitis, an antinociceptive, and an antipyretic. ${ }^{910}$ Physalins are modified steroids isolated from Physalis species with suppressive activities on macrophage and lymphocyte cultures in vitro and inhibit the production of proinflammatory mediators such as TNF. ${ }^{11,12}$ These molecules also prevent mortality induced by a lethal injection of lipopolysaccharide

\footnotetext{
* Corresponding author. Tel: +55 713176 2260. Fax: +55 713176 2272. E-mail: milena@bahia.fiocruz.br.

Centro de Pesquisas Gonçalo Moniz, Fundação Oswaldo Cruz, Salvador.

$\ddagger$ Universidade Federal de Minas Gerais, Belo Horizonte.

${ }^{\S}$ FarManguinhos, Fundação Oswaldo Cruz, Rio de Janeiro.

${ }^{\perp}$ Hospital São Rafael, Salvador.
}

$(\text { LPS })^{11}$ and inhibit rejection of allogeneic transplants in mice. ${ }^{12}$ In addition, physalins have other potent anti-inflammatory activities, decreasing injuries following intestinal ischemia and reperfusion in mice. ${ }^{13}$ This activity of physalins in the intestinal ischemia and reperfusion model, but not in in vitro assays, was blocked by the GR antagonist RU486. ${ }^{11-13}$ In the present study, the effects of physalin F (1) in a collagen-induced arthritis and allergic airway inflammation model in mice were determined and compared to the effects of dexamethasone, a gold standard glucocorticoid.

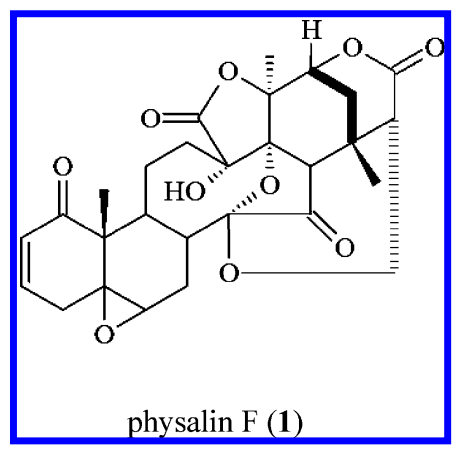

\section{Results and Discussion}

To test the effects of physalin F (1) in a model of collageninduced arthritis, mice were treated by the oral route after the onset of paw edema. A significant decrease in paw edema was observed in mice 20 days after being treated with $\mathbf{1}$ (Figure 1). Mice treated with a standard drug, dexamethasone, exhibited a significant reduction in paw edema compared to vehicle-treated mice starting 10 days after treatment (Figure 1). Twenty days after treatment, the macroscopic evaluation of paws from vehicle-treated mice showed swelling of the footpad and toes, whereas paws from 1and dexamethasone-treated mice appeared normal (Figure S1, Supporting Information).

Histological evaluation performed 20 days after treatment showed an inflammatory infiltrate of neutrophils into the articular space and in the synovial tissue, with the formation of granulation tissue and the presence of fibrin, as well as edema and congestion of vessels, in paws of all vehicle-treated mice (Figure S1, Supporting Information). In the group treated with $\mathbf{1}$, one mouse developed 


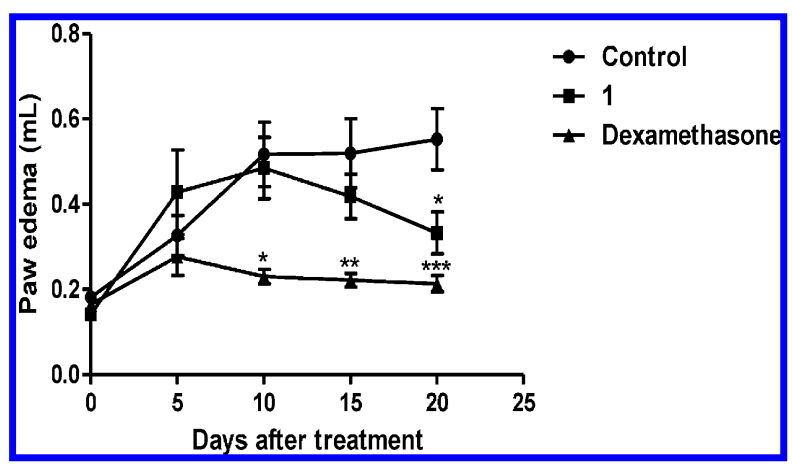

Figure 1. Treatment with physalin $F(\mathbf{1})$ in mice with collageninduced arthritis (CIA). Measurement of paw volume of DBA/1 mice with CIA. Mice were immunized with type II collagen and daily treated by oral route with vehicle, $\mathbf{1}$, or dexamethasone for 20 days. The paw volumes were measured every five days using a plethysmometer. Values are expressed as means \pm SEM of 6 or 7 mice per group, in one of two experiments performed. $(* p<0.05$; $* * p<0.01 ; * * * p<0.001$ compared to the control group).

synovial cell hyperplasia and the presence of fibrotic tissue and the deposition of fibrin in the interarticular space. The other mice in the group treated with $\mathbf{1}$ and all the mice treated with dexamethasone had paws with intact joint space, normal synovial tissue, and preserved cartilage (Figure S1, Supporting Information).

Previous work by our group has shown that $\mathbf{1}$ inhibits macrophage activation and nitric oxide production and prevents death induced by a lethal dose of LPS. ${ }^{10}$ A similar effect was found with physalin $\mathrm{B}$, which also inhibited the secretion of pro-inflammatory cytokines, such as TNF- $\alpha$, which is a pivotal cytokine in collageninduced and rheumatoid arthritis. ${ }^{10}$ In addition, physalin F suppressed the activation and proliferation of T lymphocytes. ${ }^{11}$ Thus, the beneficial effects of $\mathbf{1}$ in the model of collagen-induced arthritis may be explained by its action in both macrophages and $\mathrm{T}$ lymphocytes, which play important roles in the pathogenesis of arthritis.

The effects of treatment with $\mathbf{1}$ in lung inflammation were evaluated by comparing bronchoalveolar lavage fluid cytology of mice treated with 1 to those of mice treated with vehicle. The total number of cells (Figure 2B), the ratio of eosinophils per 200 cells (Figure $2 \mathrm{~A}$ ), and the absolute number of eosinophils (not shown) in the bronchoalveolar lavage fluid were not reduced after treatment with 1 compared to vehicle-treated mice. A significant inhibition was observed in mice treated with dexamethasone. To further characterize the changes in the lung caused by antigen challenge of immunized mice, lung sections were examined after hematoxylin and eosin staining. Ovalbumin challenge caused an intense cell infiltrate containing many lymphocytes, macrophages, and eosinophils. Mice treated with $\mathbf{1}$ exhibited a discrete reduction in lung inflammation, whereas dexamethasone had very potent inhibitory effects (Figure 2C). Lung sections from control mice revealed discrete alcian blue staining in the respiratory epithelium compared to the saline-treated group. Treatment with 1 at $60 \mathrm{mg} / \mathrm{kg}$ was not capable of modulating mucus production, since it did not decrease the number of alcian blue ${ }^{+}$cells in the lung compared to salinetreated mice (Figure 2D). The injection of the mice with allergic airway inflammation with $60 \mathrm{mg} / \mathrm{kg}$ of 1 by the intraperitoneal route (which leads to an amount of circulating drug that is higher than that obtained by oral administration) also did not lead to any protective effect (unpublished data).

The production of Th2 cytokines was quantified in the bronchoalveolar lavage fluid of individual mice from each group. As expected, the concentrations of IL- 4 and IL-13 were increased in ovalbumin-immunized mice, when compared to naive mice. Treatment with 1 did not cause a reduction in the concentrations of Th2associated cytokines in BAL fluid, which were similar to those of the saline-treated group. In contrast, dexamethasone treatment caused a marked reduction in IL-4 and IL-13 production (Figure S2, Supporting Information).

Physalin B, another steroid isolated from $P$. angulata with immunosuppressive activities, also did not decrease inflammation in this model (data not shown). The lack of anti-inflammatory activity by the physalins was associated with a lack of inhibition of Th2-associated cytokines crucial for the development of this pathological response. The treatment protocol consisted of five daily oral doses of $60 \mathrm{mg} / \mathrm{kg}$ of 1 , with each dose preceding the allergenic challenge, a fact that should favor the unveiling of any beneficial effect. This daily dose was indeed 3-fold higher than the daily dose that effectively reduced joint inflammation. It seems, therefore, that, while 1 has a potential therapeutic effect on experimental arthritis, it does not have a significant effect on experimental respiratory allergy, even following a protocol that would be better than the one usually used to treat asthmatic patients with oral corticosteroids (in which the drugs are usually given after the onset of the asthmatic crisis). It is possible, therefore, that $\mathbf{1}$ has a preferential effect on both innate, macrophage-mediated immune responses and on Th1mediated immune responses, when contrasted with corticosteroids, which would also readily inhibit Th2-mediated immune responses.

Previous studies by our group have shown that the in vitro inhibitory activities of physalins $\mathrm{B}$ and $\mathrm{F}$ on macrophage and lymphocyte activation are not blocked by the glucocorticoid receptor antagonist RU486. ${ }^{11,12}$ In contrast, RU486 administration in mice treated with the physalins blocked their protective effects in the development of ischemia and reperfusion injury in an experimental model. ${ }^{13}$ These apparently conflicting results motivated the present investigation on whether $\mathbf{1}$ activated the glucocorticoid receptor (GR), and a nuclear translocation assay of GR was performed. COS-7 cells transfected with a plasmid coding for a YFP-GR fusion protein had YFP staining mainly in the cytoplasm (Figure 3A). Transfected cells treated with dexamethasone exhibited YFP staining in the nuclei, indicating GR activation and translocation (Figure 3B). In contrast, treatment with 1 in a concentration chosen in accordance to the biological activity in vitro did not induce translocation of YFP-GR from the cytoplasm to the nucleus (Figure 3C).

The inhibitory activity of RU486 on the effects of physalins on ischemia and reperfusion injury ${ }^{13}$ can be explained by a possible dependency of the physalins on endogenous corticoids to produce those particular effects, so that the blockage of the glucocorticoid receptor by RU486 would render the animals more susceptible. The lack of action of physalins by activation of the glucocorticoid receptor was confirmed in the present work by showing that $\mathbf{1}$ did not suppress inflammation in the allergic airway inflammation model, in which the glucocorticoid receptor is known to act. In addition, 1 failed to induce the nuclear translocation of the glucocorticoid receptor in vitro, as described above, which is required for its action as a transcription factor.

A recent report has shown that physalins $\mathrm{B}$ and $\mathrm{F}$ isolated from Witheringia solanacea inhibit the activation of $\mathrm{NF}-\kappa \mathrm{B}$, a key transcription factor regulating immune-inflammatory responses. ${ }^{14}$ Vandenberghe et al. suggested that physalin B acts as an inhibitor of the ubiquitin-proteasome pathway. ${ }^{15}$ These authors showed that this inhibition may produce an accumulation of ubiquitinated proteins and consequently inhibit NF- $\kappa \mathrm{B}$ activation induced by TNF. ${ }^{15}$ Thereby it is possible that the immunosuppressive effects of physalins $\mathrm{B}$ and $\mathrm{F}$ are at least partly dependent on this mechanism of action.

The fact that physalins B and F exert their inhibitory action by a different mechanism from that of glucocorticoids may be of relevance, since the long-term use of glucocorticoids in chronic diseases such as reumathoid arthritis has several undesired side effects that perhaps 1 may lack. ${ }^{1}$ The present results also reinforce the importance of screening natural products with the particular aim of developing new immunomodulatory agents. 


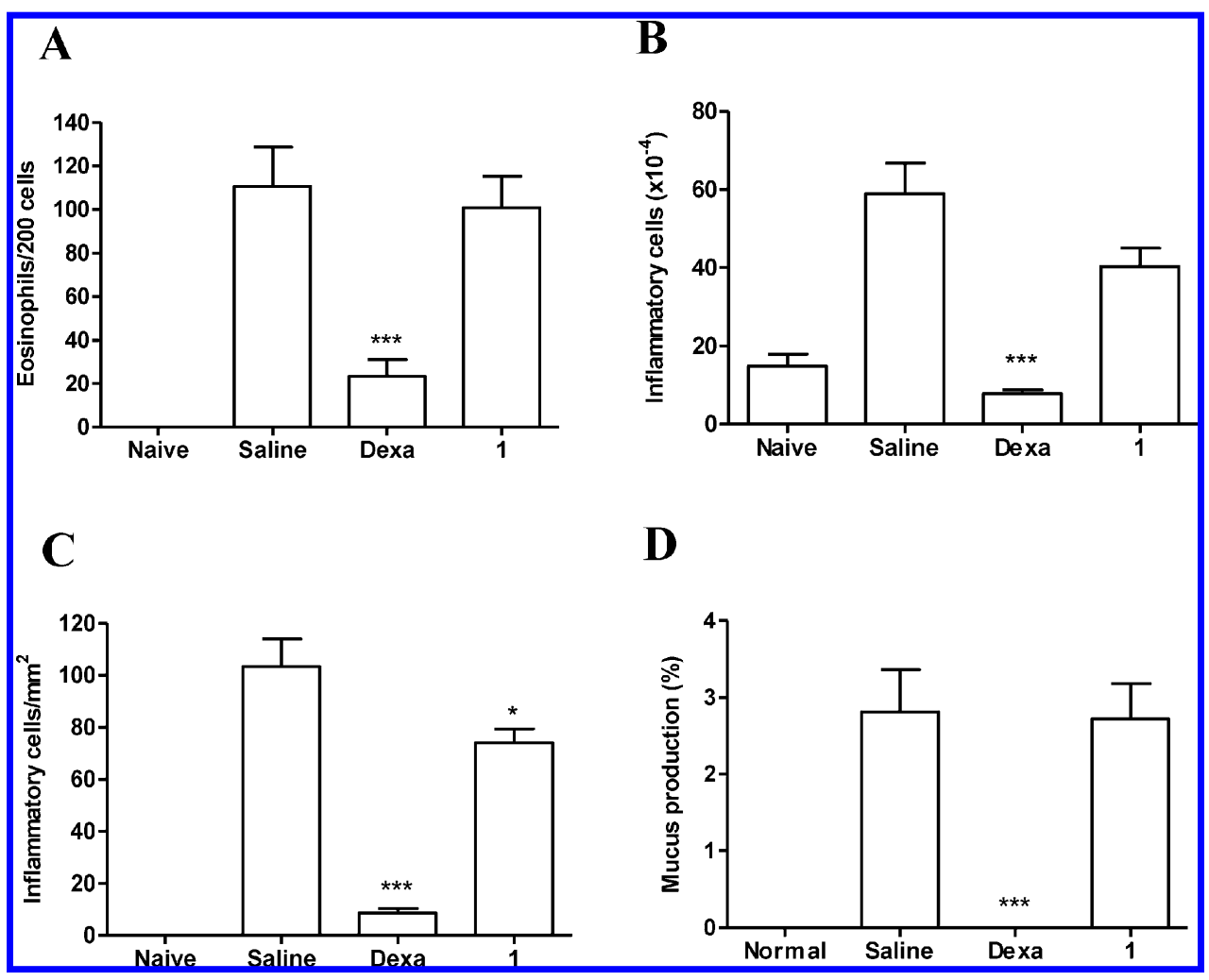

Figure 2. Effects of physalin F (1) treatment in mice with allergic airway inflammation. Mice were sacrificed $24 \mathrm{~h}$ after the last challenge with ovalbumin. The cellularity in BAL fluid and lung morphometric analysis from naive or ovalbumin-challenged mice treated with saline, dexamethasone (Dexa), or 1 were evaluated. (A) Number of eosinophils in 200 cells. (B) Total cell counts. (C) Intensity of inflammation on hematoxylin- and eosin-stained lung sections. (D) Analysis of mucus production on alcian blue-stained lung sections. Values are expressed as means \pm SEM of 6 or 7 mice per group, in one of two experiments performed $(* p<0.05 ; * * *<0.001$ compared to the control group).

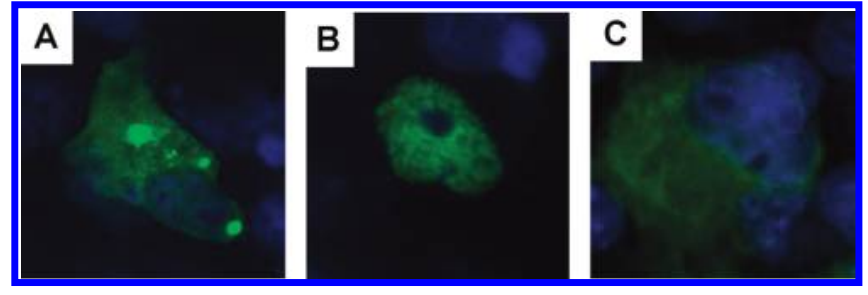

Figure 3. Physalin F (1) does not activate the nuclear translocation of the glucocorticoid receptor. Nuclear translocation assay of glucocorticoid receptor was performed in COS-7 cells transfected with a plasmid coding for an YFP-GR $\alpha$ fusion protein. Transfected cell cultures untreated (A) or treated with dexamethasone $(\mathrm{B})$ or $\mathbf{1}$ (C) were observed in a confocal microscope to analyze the YFP-GR $\alpha$ staining (yellow). Nuclei were stained with DAPI (blue).

\section{Experimental Section}

General Experimental Procedures. Male, 9- to 11-week-old DBA/1 mice were used for induction of collagen-induced arthritis. Male $\mathrm{BALB} / \mathrm{c}$ mice, 4- to 6-weeks old, were used in the allergic airway inflammation study. All mice were raised and maintained at the animal facilities at Gonçalo Moniz Research Center, FIOCRUZ (Salvador, Brazil), in rooms with controlled temperature $\left(22 \pm 2{ }^{\circ} \mathrm{C}\right)$ and humidity $(55 \pm 10 \%)$ and continuous air renovation. Animals were housed in a $12 \mathrm{~h} \mathrm{light} / 12 \mathrm{~h}$ dark cycle (6 A.M. to 6 P.M.) and provided with rodent diet and water ad libitum. Animals were handled according to the NIH guidelines for animal experimentation. All procedures described herein had prior approval from the local animal ethics committee.

Extraction and Isolation. Physalin F (1) was isolated from the stems of Physalis angulata L. collected in December 2006 in Belém do Pará, Brazil. The plant was identified by Dr. Lucia Carvalho from the Botanical Garden of Rio de Janeiro. A voucher specimen is held under number RFA 23907/8 at the Federal University of Rio de Janeiro,
Brazil, as described previously. ${ }^{12}$ The procedure used for purification used has been described. ${ }^{16,17}$ The purity of the compound was greater than $97 \%$. The sample of $\mathbf{1}$ was dissolved in dimethyl sulfoxide (DMSO) and diluted in culture medium or saline for use in the assays constituting the present study.

Induction of Collagen-Induced Arthritis and Treatment. Mice were randomized into groups of six and immunized by intradermal injection at the base of the tail with $100 \mu \mathrm{g}$ /mouse of bovine type II collagen (Chondrex, Redmond, WA), in complete Freund's adjuvant (CFA; Sigma, St Louis, MO). After an interval of 20 days, the animals received another injection of $100 \mu \mathrm{g} /$ mouse of collagen in CFA. The mice were evaluated every five days by macroscopic observation and measurement of the footpad volume using a plethysmometer (Ugo Basile, Comerio, Italy). After the appearance of swelling in the joints, approximately 10 days after the second injection of collagen, the animals were treated daily by gavage with $20 \mathrm{mg} / \mathrm{kg}$ of $1,2.5 \mathrm{mg} / \mathrm{kg}$ of dexamethasone (Sigma), or vehicle (10\% DMSO in saline), orally, for 20 days.

Sensitization and Challenge with Ovalbumin and Treatment. Allergic airway inflammation was induced as described before. ${ }^{5}$ Groups of seven mice received systemic immunization by subcutaneous injection of $10 \mu \mathrm{g}$ of chicken egg ovalbumin (Grade V, >98\% pure; Sigma, St Louis, MO) adsorbed on $50 \mu \mathrm{L}$ of $2 \mathrm{mg} / \mathrm{mL}$ alum (AlumImject; Pierce, Rockford, IL) followed by a booster injection at day 14. A nasal challenge was performed starting at day 28 , by inhalational exposure to aerosolized ovalbumin for $15 \mathrm{~min} /$ day, on five consecutive days. Exposures were carried out in an acrylic box. A solution of $1 \%$ ovalbumin in saline was aerosolized by delivery of compressed air to a sidestream jet nebulizer (Respiramax NS, São Paulo, Brazil). Two hours before each aerosol delivery, mice were treated orally with $1(60 \mathrm{mg} / \mathrm{kg})$, dexamethasone $(30 \mathrm{mg} / \mathrm{kg})$, or vehicle $(10 \%$ DMSO in saline).

Collection of Blood and Bronchoalveolar Lavage. Twenty-four hours after the last inhalational exposure, mice were sacrificed by a lethal dose of thiopental (Thiopentax; Cristália, São Paulo, Brazil). Bronchoalveolar lavage (BAL) was performed twice by intratracheal 
instillation of $1 \mathrm{~mL}$ of $0.15 \mathrm{M}$ phosphate-buffered saline, $\mathrm{pH} 7.2$ (PBS). The first lavage fluid was centrifuged, and aliquots of the supernatant were kept at $-70{ }^{\circ} \mathrm{C}$ until use for cytokine measurements. The cell pellets from the two lavages were pooled by resuspension in $1 \mathrm{~mL}$ of PBS, and the number of total leukocytes in bronchoalveolar lavage fluid was estimated in a Neubauer chamber. A differential count of 200 cells was made in a blind fashion and according to standard morphologic criteria using panotic-stained cytospin preparations.

Histopathological and Morphometric Analyses. In the collageninduced arthritis model, the animals were sacrificed with a lethal injection of barbituric anesthetic (Thiopentax; Cristália). The hind footpads were removed, fixed in $10 \%$ formalin for $48 \mathrm{~h}$, and subsequently decalcified in $7 \%$ nitric acid for 3 days, in a mechanical shaker. For the removal of excess acid, the legs were washed for 5 days with distilled water, and then sections including joints were dehydrated in graded alcohol, clarified in xylene, and embedded in paraffin. Sections of $4-6 \mu \mathrm{m}$ thick were obtained and stained with hematoxylin and eosin. For the allergic airway inflammation model, the right lobe of the lungs from each animal was removed for histological analysis. The lung was inflated via the tracheal cannula with $4 \%$ buffered formalin, fixed in the same solution, and routinely processed so as to be embedded in paraffin. Sections were stained with hematoxylin and eosin for quantification of inflammatory cells by optical microscopy. For each lung, 10 fields $(400 \times)$ were analyzed per section, and the data used to calculate the mean number of cells per $\mathrm{mm}^{2}$. Mucus production was analyzed in alcian blue-stained sections. All images were digitalized using a color digital video camera (CoolSnap cf) adapted to a BX41 microscope (Olympus, Tokyo, Japan) calibrated with a reference measurement slide and were analyzed using the Image Pro image program (version 6.1; Media Cybernetics, San Diego, CA).

Measurement of Cytokines. Concentrations of interleukin (IL)-4 and IL-13 in bronchoalveolar lavage fluid were also determined by ELISA using specific antibody kits (R\&D Systems, Minnesota, MN), according to the manufacturer's instructions. Briefly, 96-well plates were coated with the capture antibody overnight and treated by incubation with block buffer at room temperature for $1 \mathrm{~h}$. Samples were added in duplicate and incubated overnight at $4{ }^{\circ} \mathrm{C}$. Biotinylated antibodies were added and plates were incubated for $2 \mathrm{~h}$ at room temperature. A half-hour incubation with streptavidin-HRP was followed by detection using 3,5, $3^{\prime}, 5^{\prime}$-tetramethylbenzidine (TMB) and $\mathrm{H}_{2} \mathrm{O}_{2}$ as substrate and read under a $450 \mathrm{~nm}$ wavelenght light beam.

Cell Culture and Transfection. COS-7 cells were grown in Dulbecco's modified Eagle's medium (DMEM) supplemented with 10\% fetal bovine serum. One day before transfection, cells were transferred to 8-well Labtek dishes $\left(2 \times 10^{4}\right.$ cells per well). Cells were transfected using lipofectamin (Invitrogen, Carlsbad, CA) with the YFP-hGR $\alpha$ expression vector $(0.2 \mu \mathrm{g}$ of plasmid DNA/well), kindly donated by Dr. John A. Cidlowski. After a 4h incubation with the lipofectamin-plasmid mixture in serum-free DMEM, cells were refed with supplemented DMEM. One day after transfection, cells were treated with dexamethasone $(1 \mu \mathrm{M}), \mathbf{1}(3.8 \mu \mathrm{M})$, or medium for $1 \mathrm{~h}$. Cells were fixed in cold acetone, and slides were prepared using Vectashield with 4,6diamidino-2-phenylindole (VectaShield Hard Set mounting medium with DAPI H-1500; Vector Laboratories, Burlingame, CA). Cells were observed by using an Olympus confocal laser scanning microscope (FV 1000 Olympus).

Statistical Analysis. Results were expressed as means \pm SEM of 6 or 7 mice per group. Differences between groups in the collageninduced arthritis model were analyzed using multiple comparison test (Tukey-Kramer) and GraphPad InStat software version 3.05. For the allergic airway inflammation model, statistical comparisons between groups were performed by analysis of variance (ANOVA) followed by a Newman-Keuls multiple comparison test, using the GraphPad InStat program (Software Inc., San Diego, CA). Results were considered to be statistically significant when $p$ was $\leq 0.05$.

Acknowledgment. The authors thank Dr. R. D. Couto for assistance with statistical analysis and L. Ramires Santos for technical assistance. This work was supported by FIOCRUZ/PDTIS, CNPq, Institutos do Milênio, IMSEAR, MCT, FINEP, and RENORBIO.

Supporting Information Available: Figures S1 and S2. This material is available free of charge via the Internet at http://pubs.acs.org.

\section{References and Notes}

(1) Schimmer, B. P.; Parker, K. L. The Pharmacological Basis of Therapeutics; McGraw-Hill: New York, 2001; pp 1649-1677.

(2) Bosscher, K. D.; Beck, I. M.; Haegeman, G. Brain Behav. Immun. 2010, in press.

(3) Verpoorte, R.; van der Heijden, R.; Memelink, J. Transgenic Res. 2000, 9, 323-343.

(4) Basso, L. A.; da Silva, L. H.; Fett-Neto, A. G.; de Azevedo, W. F., Jr.; Moreira, I. S.; Palma, M. S.; Calixto, J. B.; Astolfi Filho, S.; Ribeiro-dos-Santos, R.; Soares, M. B. P.; Santos, D. S. Mem. Inst. Oswaldo Cruz 2005, 100, 475-506.

(5) Vasconcelos, J. F.; Teixeira, M. M.; Barbosa-Filho, J. M.; Lúcio, A. S. S. C.; Almeida, J. R. G. S.; de Queiroz, L. P.; Ribeiro-dosSantos, R.; Soares, M. B. P. Int. Immunopharmacol. 2008, 8, 12161221.

(6) Vasconcelos, J. F.; Teixeira, M. M.; Barbosa-Filho, J. M.; Agra, M. F.; Nunes, X. P.; Giulietti, A. M.; Ribeiro-dos-Santos, R.; Soares, M. B. P. Eur. J. Pharmacol. 2009, 609, 126-131.

(7) Kang, S. Y.; Yoon, S. Y.; Roh, D. H.; Jeon, M. J.; Seo, H. S.; Uh, D. K.; Kwon, Y. B.; Kim, H. W.; Han, H. J.; Lee, H. J.; Lee, J. H. J. Pharm. Pharmacol. 2008, 60, 1347-1354.

(8) Wang, C.; Dai, Y.; Yang, J.; Chou, G.; Wang, C.; Ang, Z. J. Ethnopharmacol. 2007, 111, 322-328.

(9) Lin, Y. S.; Chiang, H. C.; Kan, W. S.; Hone, E.; Shih, S. J.; Won, M. H. Am. J. Chin. Med. 1992, 20, 233-243.

(10) Bastos, G. N. T.; Silveira, A. J. A.; Salgado, C. G.; Picanço-Diniz, D. L. W.; do Nascimento, J. L. M. J. Ethnopharmacol. 2008, 118, 246-251.

(11) Soares, M. B. P.; Bellintani, M. C.; Ribeiro, I. M.; Tomassini, T. C. B.; Ribeiro-dos-Santos, R. Eur. J. Pharmacol. 2003, 459, 107-112.

(12) Soares, M. B. P.; Brustolim, D.; Santos, L. A.; Bellintani, M. C.; Paiva, F. P.; Ribeiro, Y. M.; Tomassini, T. C. B.; Ribeiro-dos-Santos, R. Int. Immunopharmacol. 2006, 6, 408-414.

(13) Vieira, A. T.; Pinho, V.; Lepsch, L. B.; Scavone, C.; Ribeiro, I. M.; Tomassini, T.; Ribeiro-dos-Santos, R.; Soares, M. B.; Teixeira, M. M.; Souza, D. G. Br. J. Pharmacol. 2005, 146, 244-251.

(14) Jacobo-Herrera, N. J.; Bremner, P.; Marquez, N.; Gupta, M. P.; Gibbons, S.; Muñoz, E.; Heinrich, M. J. Nat. Prod. 2006, 69, 328331.

(15) Vandenberghe, I.; Créancier, L.; Vispé, S.; Annereau, J. P.; Barret, J. M.; Pouny, I.; Samson, A.; Aussagues, Y.; Massiot, G.; Ausseil, F.; Bailly, C.; Kruczynski, A. Biochem. Pharmacol. 2008, 76, 453462.

(16) Tomassini, T. C. B.; Xavier, D. C. D.; Fernandez, E. F.; Ribeiro, I. M.; Soares, M. B. P.; Barbi, N. S.; Soares, R. A. O.; Ribeiro-dos-Santos, R. Brazilian Patent BRPI 9,904,635-0, 1999.

(17) Tomassini, T. C. B.; Ribeiro-dos-Santos, R.; Soares, M. B. P.; Xavier, D. C. D.; Barbi, N. S.; Ribeiro, I. M.; Soares, R. A. O.; Ferreira, E. F. U.S. Patent 6,998,394 B2, 2006.

NP900691W 